\title{
Validation of an accelerated high-sensitivity troponin T assay protocol in an Australian cohort with chest pain
}

\section{William A Parsonage \\ DM, MRCP, FRACP \\ Cardiologist \\ Jaimi H Greenslade BPsych(Hons), PhD Research Fellow Emergency Medicine \\ Christopher Hammet MB ChB, FRACP \\ Cardiologist \\ Arvin Lamanna MB BS, FRACP Cardiology Registrar \\ Jillian R Tate BSc(Hons), MSc Scientist, Chemical Pathology \\ Jacobus P Ungere MB ChB, MMed, FRCPA Director, Chemical Pathology \\ Kevin Chu MB BS, MSC Emergency Physician \\ Martin Than MB BS \\ Emergency Physician \\ Anthony F T Brown MB ChB, Emergency Physician \\ Louise Cullen MB BS, Emergency Physician \\ Royal Brisbane an Women's Hospita Brisbane, QLD. \\ 2 Christchurch Hospital, Christchurch, New Zealand. \\ william_parsonage@ health.qld.gov.au}

MJA 2014; 200: 161-165 doi: 10.5694/mjal3.10466
(1) levation of cardiac troponin (cTn) levels is central to the definition of acute myocardial infarction (AMI). ${ }^{1}$ Increasingly sensitive cTn assays offer the potential for AMI to be diagnosed earlier than the time points recommended in established clinical guidelines. ${ }^{2}$ Earlier diagnosis allows for more timely initiation of therapy for those with AMI and earlier rule-out of AMI for others. This is relevant to overcrowded hospital emergency departments, where chest pain remains one of the most common reasons for presentation. ${ }^{3}$ In Australia, time-based political imperatives such as the National Emergency Access Target have further increased the need for efficient yet safe means of assessing patients with chest pain. ${ }^{4}$

No universally agreed nomenclature of cTn assays exists. It is generally held that a highly sensitive assay should have a coefficient of variation (CV) of $<10 \%$ at the level of the 99th percentile of a healthy population and should be able to measure cTn in $>50 \%$ of such a population. Recent guidance includes preliminary advice regarding the appropriate use of highly sensitive assays. ${ }^{2}$ One such assay has been in clinical use in Australia since 2011 and although its uptake has been brisk, a corresponding change in the diagnostic approach according to this guidance has been variable. The reasons for this are unknown but may include the reference to a diagnostic cut-off that is at odds with the universal definition of myocardial infarction, ${ }^{5,6}$ reluctance to change long established practice, or limited local clinical outcome data using this assay.

Our primary aim was to examine the diagnostic accuracy of the high-sensitivity cardiac troponin $\mathrm{T}$ (hs-cTnT) assay for the diagnosis of AMI, using serial measurements at $0 \mathrm{~h}$ and $2 \mathrm{~h}$ after presentation, in an unselected, well characterised cohort of patients with chest pain. Our secondary aim

\section{Abstract}

Objectives: To validate an accelerated biomarker strategy using a highsensitivity cardiac troponin T (hs-cTnT) assay for diagnosing acute myocardial infarction (AMI) in patients presenting to the emergency department with chest pain; and to validate this strategy in combination with the National Heart Foundation of Australia/Cardiac Society of Australia and New Zealand risk stratification model.

Design, setting and patients: Single-centre, prospective, observational cohort study of 764 adults presenting to a tertiary hospital with symptoms of possible acute coronary syndrome between November 2008 and February 2011.

Main outcome measures: AMI or cardiac death within 24 hours of presentation (primary), and major adverse cardiac events within 30 days (secondary).

Results: An elevated hs-cTnT assay result above the 99th percentile at either the $\mathrm{O}$ h or $2 \mathrm{~h}$ time points had sensitivity of $96.4 \%(95 \% \mathrm{Cl}, 87.9 \%-99.0 \%)$, specificity of $82.6 \%(95 \% \mathrm{Cl}, 79.7 \%-85.2 \%)$, negative predictive value of $99.7 \%(95 \% \mathrm{Cl}, 98.8 \%-99.9 \%)$ and positive predictive value of 30.5\% (95\% $\mathrm{Cl}, 24.2 \%-37.6 \%$ ) for diagnosing AMI. Compared with a traditional $6 \mathrm{~h}$ cardiac troponin testing strategy, the accelerated strategy led to reclassification of risk in only two patients with adverse cardiac outcomes, with no net effect on appropriate management.

Conclusions: In patients presenting with chest pain, an accelerated biomarker strategy using the hs-cTnT assay performed well in the initial diagnosis of AMI. The accelerated strategy was also effective when incorporated into a comprehensive strategy of risk stratification that included clinical and demographic factors. The time saved by this approach could have a major impact on health service delivery.

Trial registration: Australian New Zealand Clinical Trials Registry ACTRN12610000053022.

was to examine the accuracy of predicting 30-day major adverse cardiac events (MACE) using a model incorporating $0 \mathrm{~h}$ and $2 \mathrm{~h}$ hs-cTnT assay measurements and risk stratification according to the guidelines of the National Heart Foundation of Australia and Cardiac Society of Australia and New Zealand (NHFA/CSANZ). ${ }^{7}$

\section{Methods}

This was a prespecified substudy of a prospective, observational cohort study conducted between November 2008 and February 2011. Consecutive eligible patients presenting during office hours to a single, large, metropolitan tertiary hospital emergency department with symptoms suggestive of cardiac chest pain were recruited. Inclusion criteria were age $\geqslant 18$ years, and at least 5 minutes of possible cardiac symptoms where the attending physician intended to perform serial cTn tests. We used Ameri- can Heart Association definitions for possible cardiac symptoms (ie, acute chest, epigastric, neck, jaw or arm pain; or discomfort or pressure without an apparent non-cardiac source). ${ }^{8}$ Patients were excluded for any of the following: a clear cause of symptoms other than acute coronary syndrome (ACS); inability or unwillingness to provide consent or be contacted after discharge; recruitment considered inappropriate by staff (eg, palliative treatment); interhospital transfer; pregnancy; and previous enrolment. Perceived high risk of ACS was not an exclusion criterion. Patients with missing blood samples were also excluded. The study was carried out according to the principles of the Declaration of Helsinki, approved by the Royal Brisbane and Women's Hospital Human Research Ethics Committee (2008/ 101), and registered with the Australian New Zealand Clinical Trials Registry (ACTRN12610000053022). Written informed consent was obtained. 


\begin{tabular}{lc} 
1 Demographic characteristics of the cohort & \\
Characteristic & Total $(n=764) *$ \\
\hline Mean (SD) age (years) & $55.3(15.1)$ \\
Male & $468(61.3 \%)$ \\
Risk factors & \\
Hypertension & $376(49.2 \%)$ \\
Diabetes & $112(14.7 \%)$ \\
Dyslipidaemia & $389(50.9 \%)$ \\
Family history & $386(50.5 \%)$ \\
Smoking (recent or current) & $237(31.0 \%)$ \\
Prior medical history & $192(25.1 \%)$ \\
Angina & $137(17.9 \%)$ \\
Acute myocardial infarction & $49(6.4 \%)$ \\
Coronary artery bypass grafting & $82(10.7 \%)$ \\
Percutaneous coronary intervention & $4.97(1.63-20.60)$ \\
Median (IQR) time from first symptoms to & \\
presentation to hospital (h) & $74.1(184.8)$ \\
Hospital length of stay (h) & $28.0(10.2-75.4)$ \\
Mean (SD) & \\
Median (IQR) & \\
\hline
\end{tabular}

IQR = interquartile range. * Unless otherwise specified, data are number (\% of whole cohort).

\section{Frequency of 30-day major adverse cardiac events (MACE)}

\begin{tabular}{lccc} 
Event & No.* & $\begin{array}{c}\text { Frequency in } \\
\text { patients with MACE } \\
(n=83)\end{array}$ & $\begin{array}{c}\text { Frequency in } \\
\text { whole cohort } \\
(n=764)\end{array}$ \\
\hline Cardiac death & 2 & $2.4 \%$ & $0.3 \%$ \\
Cardiogenic shock & 2 & $2.4 \%$ & $0.3 \%$ \\
STEMI & 17 & $20.5 \%$ & $2.2 \%$ \\
NSTEMI & 43 & $51.8 \%$ & $5.6 \%$ \\
UAP & 24 & $28.9 \%$ & $3.1 \%$ \\
Revascularisation & & & \\
Emergency & 18 & $21.7 \%$ & $2.4 \%$ \\
Urgent & 31 & $37.3 \%$ & $4.1 \%$ \\
Elective & 4 & $4.8 \%$ & $0.5 \%$ \\
\hline
\end{tabular}

STEMI = ST-elevation myocardial infarction. NSTEMI = non-ST-elevation myocardial infarction. UAP = unstable angina pectoris. *A total of 141 events were observed in 83 patients.

\section{Outcomes}

The primary outcome was a composite of AMI and cardiac death within $24 \mathrm{~h}$ of presentation. The diagnostic accuracy of the hs-cTnT assay at $0 \mathrm{~h}$, $2 \mathrm{~h}$ and $0 \mathrm{~h}$ or $2 \mathrm{~h}$ was compared with adjudicated diagnoses based on all other clinical data.

The secondary outcome was MACE within 30 days of presentation (including AMI and cardiac deaths within $24 \mathrm{~h}$ ). The secondary analysis assessed the predictive performance of NHFA/CSANZ clinical risk stratification ${ }^{7}$ when incorporating the hs-cTnT assay at $0 \mathrm{~h}$ and $2 \mathrm{~h}$, compared with the conventional approach of measuring cTn at $0 \mathrm{~h}$ and $6-8 \mathrm{~h}$. MACE was a composite of cardiac death, ACS (encompassing ST-segment-elevation myocardial infarction [STEMI], non-ST-segment-elevation myocardial infarction [NSTEMI] and unstable angina pectoris [UAP]) and coronary revascularisation.

\section{Routine clinical assessment}

Patients were managed according to local hospital protocols. Our institution uses the Queensland Health Cardiac Chest Pain Risk Stratification Pathway, ${ }^{9}$ which is based on the NHFA/CSANZ guidelines.

\section{Investigational hs-cTnT assay}

Blood samples for analysis by the hscTnT assay (Roche Diagnostics) were collected in serum tubes at presentation and then $2 \mathrm{~h}$ afterwards. After centrifugation, samples were frozen at $-80^{\circ} \mathrm{C}$ until assayed blind using the Cobas e601 immunology analyser (Roche Diagnostics) in a core laboratory. The limit of blank (LoB) and limit of detection (LoD) were $3 \mathrm{ng} / \mathrm{L}$ and $5 \mathrm{ng} / \mathrm{L}$, respectively. The imprecision of the assay gave a $10 \% \mathrm{CV}$ at a level of $13 \mathrm{ng} / \mathrm{L}$, and the 99th percentile of a healthy reference population was $14 \mathrm{ng} / \mathrm{L}$. Advice received from the manufacturer after the initial assay indicated that measurements had been performed with reagent lots using a calibration curve suboptimally standardised in the low range. Results were recalculated by the manufacturer with a restandardised calibration curve for the reagent kit. This revision was performed blind to the clinical end points. We used the revised values for all study analyses.

\section{Adjudication of end points}

For adjudicated diagnoses, AMI was defined as recommended in current guidelines using all available clinical data acquired during routine management. ${ }^{1}$ Final diagnoses were adjudicated independently by one of two cardiologists, with all ACS end points and $10 \%$ of non-ACS end points readjudicated by both cardiologists. Consensus was achieved for all end points.

As per hospital protocol, blood was drawn for local cardiac troponin I (cTnI) measurement at presentation and then $6 \mathrm{~h}$ afterwards. The cTnI values, measured with the Access Accu-cTnI assay on a UniCel DxI 800 platform (Beckman Coulter), were used for adjudication. This assay had an $\mathrm{LoD}$ of $10 \mathrm{ng} / \mathrm{L}$, and imprecision giving a $10 \% \mathrm{CV}$ at $60 \mathrm{ng} / \mathrm{L}$. The 99 th percentile of a healthy reference population was $40 \mathrm{ng} / \mathrm{L}$, and this was used as the cut-off for myocardial necrosis. A change in cTnI of $\geqslant 20 \%$ was used to detect a changing pattern. If the cTnI level was elevated without a rising or falling pattern, other causes were considered. Results of the investigational hs-cTnT assay were not available at the time of adjudication.

\section{Statistical analysis}

Data were analysed using SPSS, version 19 (SPSS Inc). Baseline characteristics of the cohort are reported with descriptive statistics. We calculated diagnostic accuracy statistics to examine the utility of the hs-cTnT assay at $0 \mathrm{~h}, 2 \mathrm{~h}$ and $0 \mathrm{~h}$ or $2 \mathrm{~h}$ for diagnosing occurrence of AMI or cardiac death within $24 \mathrm{~h}$ of presentation. Estimates of positive and negative predictive value are based on the prevalence of disease in this study and therefore apply only to the population of patients presenting to hospital with chest pain.

To examine the utility of the hscTnT assay when used within the NHFA/CSANZ guidelines, ${ }^{7}$ patients were classified as low, intermediate or high risk according to the $0 \mathrm{~h}$ and $2 \mathrm{~h}$ hs-cTnT assay results compared with the $0 \mathrm{~h}$ and $6 \mathrm{~h}$ cTnI results. In keeping with current clinical practice regarding risk stratification, 22 patients whose initial electrocardiogram was suggestive of STEMI were excluded from this secondary analysis.

\section{Results}

There were 1495 patients who met the initial inclusion criteria. Of these, 517 were subsequently excluded and 214 did not have serial blood samples stored. Characteristics of the remaining 764 patients are shown in Box 1.

There were 141 major cardiac events in 83 patients $(10.9 \%$ of the cohort) within 30 days of presentation (Box 2).

Box 3 describes the accuracy of the hs-cTnT assay at $0 \mathrm{~h}, 2 \mathrm{~h}$ and $0 \mathrm{~h}$ or $2 \mathrm{~h}$ in diagnosing AMI within $24 \mathrm{~h}$ of presentation (there were no car- 


\begin{tabular}{|c|c|c|c|c|c|c|}
\hline \multirow[b]{2}{*}{ hs-cTnT assay } & \multirow[b]{2}{*}{$\begin{array}{l}\text { Patients with } \\
\text { primary outcome } \\
(n=56)\end{array}$} & \multirow[b]{2}{*}{$\begin{array}{l}\text { Patients without } \\
\text { primary outcome } \\
\text { (n=708) }\end{array}$} & \multicolumn{4}{|c|}{ Diagnostic accuracy } \\
\hline & & & $\begin{array}{l}\text { Sensitivity } \\
(95 \% \mathrm{Cl})\end{array}$ & $\begin{array}{l}\text { Specificity } \\
(95 \% \mathrm{Cl})\end{array}$ & $\begin{array}{c}\text { Negative } \\
\text { predictive value } \\
(95 \% \mathrm{Cl})\end{array}$ & $\begin{array}{c}\text { Positive } \\
\text { predictive value } \\
(95 \% \mathrm{Cl})\end{array}$ \\
\hline \multicolumn{7}{|l|}{$\mathrm{Oh}$} \\
\hline Positive (> 14 ng/L) & $52(92.9 \%)$ & $113(16.0 \%)$ & \multirow{2}{*}{$\begin{array}{c}92.9 \% \\
(83.0 \%-97.2 \%)\end{array}$} & \multirow{2}{*}{$\begin{array}{c}84.0 \% \\
(81.2 \%-86 \%)\end{array}$} & \multirow{2}{*}{$\begin{array}{c}99.3 \% \\
(98.3 \%-99.7 \%)\end{array}$} & \multirow{2}{*}{$\begin{array}{c}31.5 \% \\
(24.9 \%-39.0 \%)\end{array}$} \\
\hline Negative ( $\leqslant 14 \mathrm{ng} / \mathrm{L}$ ) & $4(7.1 \%)$ & 595 (84.0\%) & & & & \\
\hline \multicolumn{7}{|l|}{$2 \mathrm{~h}$} \\
\hline Positive (> 14 ng/L) & $54(96.4 \%)$ & $116(16.4 \%)$ & \multirow{2}{*}{$\begin{array}{c}96.4 \% \\
(87.9 \%-99.0 \%)\end{array}$} & \multirow{2}{*}{$\begin{array}{c}83.6 \% \\
(80.7 \%-86.2 \%)\end{array}$} & \multirow{2}{*}{$\begin{array}{c}99.7 \% \\
(98.8 \%-99.9 \%)\end{array}$} & \multirow{2}{*}{$\begin{array}{c}31.8 \% \\
(25.2 \%-39.1 \%)\end{array}$} \\
\hline Negative ( $\leqslant 14 \mathrm{ng} / \mathrm{L}$ ) & $2(3.6 \%)$ & $592(83.6 \%)$ & & & & \\
\hline \multicolumn{7}{|l|}{ Either $\mathrm{Oh}$ or $2 \mathrm{~h}$} \\
\hline Positive (> 14 ng/L) & $54(96.4 \%)$ & $123(17.4 \%)$ & \multirow{2}{*}{$\begin{array}{c}96.4 \% \\
(87.9 \%-99.0 \%)\end{array}$} & \multirow{2}{*}{$\begin{array}{c}82.6 \% \\
(79.7 \%-85.2 \%)\end{array}$} & \multirow{2}{*}{$\begin{array}{c}99.7 \% \\
(98.8 \%-99.9 \%)\end{array}$} & \multirow{2}{*}{$\begin{array}{c}30.5 \% \\
(24.2 \%-37.6 \%)\end{array}$} \\
\hline Negative ( $\leqslant 14 \mathrm{ng} / \mathrm{L}$ ) & $2(3.6 \%)$ & $585(82.6 \%)$ & & & & \\
\hline
\end{tabular}

diac deaths within $24 \mathrm{~h}$ ), compared with the adjudicated end points. Compared with the reference standard using cTnI measurement at $6 \mathrm{~h}$, the hs-cTnT assay resulted in two false negative cases (Box 4). Both these patients had an adjudicated diagnosis of NSTEMI in the context of a prior history of coronary artery disease.

NHFA/CSANZ risk stratification incorporating the hs-cTnT assay at $0 \mathrm{~h}$ and $2 \mathrm{~h}$ performed similarly to the conventional strategy incorporating cTnI testing at $0 \mathrm{~h}$ and $6 \mathrm{~h}$ (Box 5). One patient with an adjudicated diagnosis of NSTEMI was classified as intermediate risk using the hs-cTnT assay but high risk using the $6 \mathrm{~h}$ cTnI assay; and one patient with UAP was classified as high risk using the hscTnT assay but intermediate risk using the $6 \mathrm{~h}$ cTnI assay (Box 6). No patients were incorrectly classified as low risk using the $2 \mathrm{~h}$ strategy when compared with the standard $6 \mathrm{~h}$ strategy.

Most of the 123 patients who had elevated hs-cTnT assay results and an adjudicated diagnosis other than AMI had some other significant cardiac diagnosis (Box 7).

\section{Discussion}

In this first analysis of the performance of an accelerated biomarker strategy using the hs-cTnT assay for diagnosing AMI in an Australian cohort of patients presenting with chest pain, we found that using the hs-cTnT assay in the $2 \mathrm{~h}$ after presentation performed well compared with the conventional approach of serial
cTn testing over $6 \mathrm{~h}$. The hs-cTnT assay is currently the only highly sensitive troponin assay in widespread clinical use in Australia. It has been widely adopted despite the lack of locally derived data regarding its diagnostic performance. Our data provide a framework in which the assay may be used for rapid exclusion of AMI, such that patients may be discharged (if otherwise considered low risk) or proceed rapidly to further testing to exclude UAP (if intermediate risk).

Exclusion of a diagnosis of AMI based on clinical grounds alone has been shown to be unsatisfactory, and discharge with a missed AMI carries a poor prognosis. ${ }^{10}$ Therefore, a period of clinical observation and serial testing has been recommended for those at intermediate risk of ACS. ${ }^{7}$ Critically, we have shown that the negative predictive value of serial measurements with the hs-cTnT assay at or below the 99th percentile in the $2 \mathrm{~h}$ after presentation excluded a diagnosis of AMI with a very high level of certainty (over 99\%).

\section{Patients with negative high-sensitivity cardiac troponin T} (hs-cTnT) assays and adjudicated diagnoses of acute myocardial infarction (AMI)

Patient 1 was a man aged in his 70s with known hypertension and dyslipidaemia, and previous AMl, angina and coronary artery bypass surgery. His cardiac troponin I (CTnl) values were $20 \mathrm{ng} / \mathrm{L}$ and $200 \mathrm{ng} / \mathrm{L}$ at $\mathrm{h}$ and $6 \mathrm{~h}$, respectively. His $\mathrm{O} \mathrm{h}$ and $2 \mathrm{~h}$ electrocardiograms (ECGs) were categorised as abnormal but not diagnostic of ischaemia. Angiography showed multivessel coronary artery disease. The adjudicated diagnosis was non-ST-segment-elevation myocardial infarction (NSTEMI). His hs-CTnT values were $11.7 \mathrm{ng} / \mathrm{L}$ and $13.3 \mathrm{ng} / \mathrm{L}$ at $\mathrm{O}$ h and $2 \mathrm{~h}$, respectively.

Patient 2 was a woman aged in her 60 s with a past history of hypertension, dyslipidaemia and coronary artery disease with angina. Her cTnl values were $10 \mathrm{ng} / \mathrm{L}$ and $200 \mathrm{ng} / \mathrm{L}$ at $O \mathrm{~h}$ and $6 \mathrm{~h}$, respectively. Her $\mathrm{O}$ and $2 \mathrm{~h}$ ECGs were categorised as normal. She had positive stress myocardial perfusion imaging, and the adjudicated diagnosis was NSTEMI. Her hs-cTnT value was $9.3 \mathrm{ng} / \mathrm{L}$ at both $\mathrm{O}$ and $2 \mathrm{~h}$.

Two patients with an adjudicated end point of NSTEMI had negative hs-cTnT assays at $0 \mathrm{~h}$ and $2 \mathrm{~h}$, yielding an overall sensitivity of $96 \%$. One patient had early objective investigation and was found to have reversible ischaemia on non-invasive testing. The other patient would have been considered at high risk of ACS due to a prior history of coronary artery bypass surgery. In keeping with current guidelines, further investigation would have been recommended for both patients. These

\section{Comparison of risk classification* and rates of $\mathrm{MACE}^{\dagger}$ within 30 days using the high-sensitivity cardiac troponin T (hs-cTnT) assay versus the cardiac troponin I (cTnl) assay}

\begin{tabular}{lcc} 
Risk category & hs-cTnT assay at O $\mathrm{h}$ and $2 \mathrm{~h}$ & cTnl assay at O h and 6h \\
\hline Low & $0 / 8(0[0-3.2 \%])$ & $0 / 9(0[0-3.0 \%])$ \\
Intermediate & $11 / 457(2.4 \%[1.3 \%-4.3 \%])$ & $11 / 478(2.3 \%[1.3 \%-4.1 \%])$ \\
High & $56 / 277(20.2 \%[15.9 \%-25.3 \%])$ & $56 / 255(22.0 \%[17.3 \%-27.4 \%])$
\end{tabular}

MACE = major adverse cardiac events. * According to National Heart Foundation of Australia and Cardiac Society of Australia and New Zealand guidelines. ${ }^{7} \dagger$ Data are number of patients with MACE/ number of patients in risk category (\% of risk category [ $95 \% \mathrm{Cl}]$ ). This analysis excludes 22 patients with an initial electrocardiogram suggestive of ST-segment-elevation myocardial infarction, for whom further risk stratification is irrelevant. 


\section{Patients with 30-day MACE and differing risk} stratification according to the high-sensitivity cardiac troponin T (hs-cTnT) assay and the cardiac troponin I (cTnl) assay

Patient 1 was a woman aged in her 60 s with a past history of hypertension, dyslipidaemia and coronary artery disease with angina. Her cTnl values were $10 \mathrm{ng} / \mathrm{L}$ and $200 \mathrm{ng} / \mathrm{L}$ at $0 \mathrm{~h}$ and $6 \mathrm{~h}$, respectively. $\mathrm{Her} \mathrm{O} \mathrm{h}$ and $2 \mathrm{~h}$ electrocardiograms were categorised as normal. She had positive stress myocardial perfusion imaging and the adjudicated diagnosis was non-ST-segment-elevation myocardial infarction. Her $\mathrm{hs}-\mathrm{cTnT}$ value was $9.3 \mathrm{ng} / \mathrm{L}$ at both $\mathrm{O} \mathrm{h}$ and $2 \mathrm{~h}$.

Patient 2 was a man aged in his 60 s with a past history of percutaneous coronary intervention ( $>6$ months before presentation), hypertension, dyslipidaemia and family history of coronary artery disease. His $\mathrm{CTnl}$ value was $10 \mathrm{ng} / \mathrm{L}$ at both $\mathrm{O} \mathrm{h}$ and $6 \mathrm{~h}$. Angiography showed two-vessel coronary artery disease. The adjudicated diagnosis was unstable angina pectoris. His hs-cTnT values were $14.3 \mathrm{ng} / \mathrm{L}$ and $12.5 \mathrm{ng} / \mathrm{L}$ at $\mathrm{O} \mathrm{h}$ and $2 \mathrm{~h}$, respectively.

$\mathrm{MACE}=$ major adverse cardiac events.

cases clearly illustrate that cardiac biomarkers alone cannot be relied on for diagnosis of patients presenting with chest pain. The results of any biomarker strategy must be interpreted in the context of a recognised systematic approach that incorporates other clinical factors.

We also found that the strategy of early serial testing with the hs-cTnT assay performed well when incorporated into the model of risk stratification for MACE recommended by the NHFA/CSANZ, ${ }^{7}$ compared with conventional serial testing over a longer period. Although more patients were considered to be at high risk using the hs-cTnT assay $(277 \mathrm{v} 255)$, the 30-day MACE rates in high-risk patients were comparable using either assay (20\% with hs-cTnT v $22 \%$ with cTnI). Only two patients with 30-day MACE were reclassified when the hs-cTnT assay was incorporated into risk stratification. Importantly, no patients classified as low risk using either assay had a major cardiac event during the 30-day follow-up period.

As expected, an increase in sensitivity of the troponin assay led to some corresponding fall in specificity. Nevertheless, most patients with an elevated hs-cTnT assay result had a significant cardiac diagnosis even in the absence of AMI. Thus, even when using a highly sensitive assay, a level above the 99th percentile is rarely insignificant.

Our study has some limitations. First, only patients presenting with chest pain were recruited, so our results cannot be extrapolated to other modes of presentation of ACS, such as dyspnoea or syncope in the absence of chest pain. Also, consecutive recruitment was performed only within office hours, but this is unlikely to have had any significant effect on our findings.

Second, an emerging concept is that the diagnostic performance of high-sensitivity troponin assays can be optimised by a more sophisticated approach to their interpretation that incorporates delta values as well as absolute cut-offs. One recent study using early biomarker testing supports such an approach to enhance the accuracy of the test. ${ }^{11}$ Although we did not address this hypothesis in our analysis, it is included in a larger multicentre study that is underway.

Finally, we did not examine the performance of the hs-cTnT assay in line with guidance regarding the appropriate timing of measurement and magnitude of delta troponin required to diagnose AMI, as suggested in the 2011 addendum to the

7 Principal adjudicated diagnoses in 123 patients with elevated high-sensitivity cardiac troponin T assay results but without a diagnosis of acute myocardial infarction (AMI)

\begin{tabular}{lccc} 
Diagnosis & No. & $\begin{array}{c}\text { Proportion of patients with non- } \\
\text { AMI diagnosis }(n=123)\end{array}$ & $\begin{array}{c}\text { Proportion of all patients } \\
(n=764)\end{array}$ \\
\hline Stable CAD or UAP & 27 & $22.0 \%$ & $3.5 \%$ \\
Atrial fibrillation or other SVT & 18 & $14.6 \%$ & $2.4 \%$ \\
Heart failure & 9 & $7.3 \%$ & $1.2 \%$ \\
Pericarditis & 5 & $4.1 \%$ & $0.7 \%$ \\
Hypotension or syncope & 4 & $3.3 \%$ & $0.5 \%$ \\
Life-threatening arrhythmia & 3 & $2.4 \%$ & $0.4 \%$ \\
Inappropriate ICD shock & 1 & $0.8 \%$ & $0.1 \%$ \\
Significant valve disease & 1 & $0.8 \%$ & $0.1 \%$ \\
Pulmonary embolus & 1 & $0.8 \%$ & $0.1 \%$ \\
Non-cardiac or other & 54 & $43.9 \%$ & $7.1 \%$ \\
\hline CAD coronary artery disease. UAP = unstable angina pectoris. SVT = supraventricular tachycardia. ICD = implantable
\end{tabular}

NHFA/CSANZ guidelines. ${ }^{2}$ Our study commenced before this publication appeared, and the decision to sample cTn at $2 \mathrm{~h}$ after presentation was based on data suggesting a high degree of accuracy of sensitive cTn assays $^{12}$ and our previous studies of accelerated diagnostic protocols for chest pain using other biomarker strategies at this time point. ${ }^{13,14}$ Additionally, the NHFA/CSANZ recommendations regarding interpretation of changing cTn levels are based largely on studies of assay performance and biological variability in healthy populations, and they await confirmation in studies based on clinical outcomes. ${ }^{15}$ Nevertheless, we cannot exclude the possibility that later blood sampling would have altered the performance of the assay in our cohort.

In summary, we have shown that, for patients presenting with chest pain, the hs-cTnT assay measured at presentation and $2 \mathrm{~h}$ later performed well in the initial diagnosis of AMI. We also found it is effective when incorporated into a comprehensive risk stratification strategy that includes clinical evaluation and early non-invasive testing for ischaemia, where appropriate. The time saved by this approach is considerable and, given the burden of patients presenting with chest pain, could have a major impact on health service delivery. Translation into improved patient outcomes or significant cost savings remains an important area for further research. However, our findings provide validation in an Australian cohort of the hs-cTnT assay that is already in widespread clinical use.

Acknowledgements: We are indebted to the patients who participated in the study. We thank the research, emergency department and laboratory staff for their valuable efforts. This study was supported by research grants from the Queensland Emergency Medicine Research Foundation (QEMRF Proj-2008-002) and Roche Diagnostics. Roche Diagnostics also supplied reagents. The study sponsors had no role in the study design, data collection, data analysis, data interpretation, or the writing of or decision to submit the report.

Competing interests: Louise Cullen, Anthony Brown, Martin Than, Jaimi Greenslade, Christopher Hammett, Jacobus Ungerer, Kevin Chu and William Parsonage were investigators on a Roche Diagnostics research grant, which provided reagents and funding to facilitate this project. Roche Diagnostics had no role in the study design or implementation or preparation of the manuscript.

Received 11 Apr 2013, accepted 22 Aug 2013.

1 Thygesen K, Alpert JS, Jaffe AS, et al. Third universal definition of myocardial infarction. Circulation 2012; 126: 2020-2035. 
2 Chew DP, Aroney CN, Aylward PE, et al. 2011 Addendum to the National Heart Foundation of Australia/Cardiac Society of Australia and New Zealand Guidelines for the management of acute coronary syndromes (ACS) 2006. Heart Lung Circ 2011; 20: 487-502.

3 Nawar EW, Niska RW, Xu J. National Hospital Ambulatory Medical Care Survey: 2005 emergency department summary. Adv Data 2007; (386): 1-32.

4 Australian Government Department of Health and Ageing. A national health and hospitals network for Australia's future - delivering better health and better hospitals. Canberra: DoHA 2010.

5 Thygesen K, Alpert JS, White HD, et al. Universal definition of myocardial infarction. Circulation 2007; 116: 2634-2653.

6 Ungerer JP, Pretorius CJ, Wilgen U, Tate JR. Ambiguous cardiac troponin recommendations. Heart Lung Circ 2012; 21: 197.

7 Acute Coronary Syndrome Guidelines Working Group Guidelines for the management of acute coronary syndromes 2006. Med J Aust 2006; 184 (8 Suppl): S1-S32.

8 Luepker RV, Apple FS, Christenson RH, et al. Case definitions for acute coronary heart disease in epidemiology and clinical research studies: a statement from the AHA Council on

Epidemiology and Prevention; AHA Statistics Committee; World Heart Federation Council on Epidemiology and Prevention; the European Society of Cardiology Working Group on

Epidemiology and Prevention; Centers for Disease Control and Prevention; and the National Heart, Lung, and Blood Institute. Circulation 2003; 108: 2543-2549.

9 Queensland Government. Emergency department cardiac chest pain risk stratification pathway. Brisbane: Queensland Health, 2012 http://www.health.qld.gov.au/caru/pathways/ docs/pathway chstpain.pdf (accessed Jan 2014).

10 Pope JH, Aufderheide TP, Ruthazer R, et al. Missed diagnoses of acute cardiac ischemia in the emergency department. N Engl J Med 2000; 342: $1163-1170$.
11 Reichlin T, Schindler C, Drexler B, et al. One-hour rule-out and rule-in of acute myocardial infarction using high-sensitivity cardiac troponin T. Arch Intern Med 2012; 172: 1211-1218.

12 Reichlin T, Hochholzer W, Bassetti S, et al. Early diagnosis of myocardial infarction with sensitive cardiac troponin assays. N Engl J Med 2009; 361: 858-867.

13 Than M, Cullen L, Reid CM, et al. A 2-h diagnostic protocol to assess patients with chest pain symptoms in the Asia-Pacific region (ASPECT): a prospective observational validation study. Lancet 2011; 377: 1077-1084.

14 Than M, Cullen L, Aldous S, et al. 2-Hour accelerated diagnostic protocol to assess patients with chest pain symptoms using contemporary troponins as the only biomarker: the ADAPT trial. J Am Coll Cardiol 2012; 59: 2091-2098.

15 Thygesen K, Mair J, Giannitsis E, et al. How to use high-sensitivity cardiac troponins in acute cardiac care. Eur Heart J 2012; 33: 2252-2257.

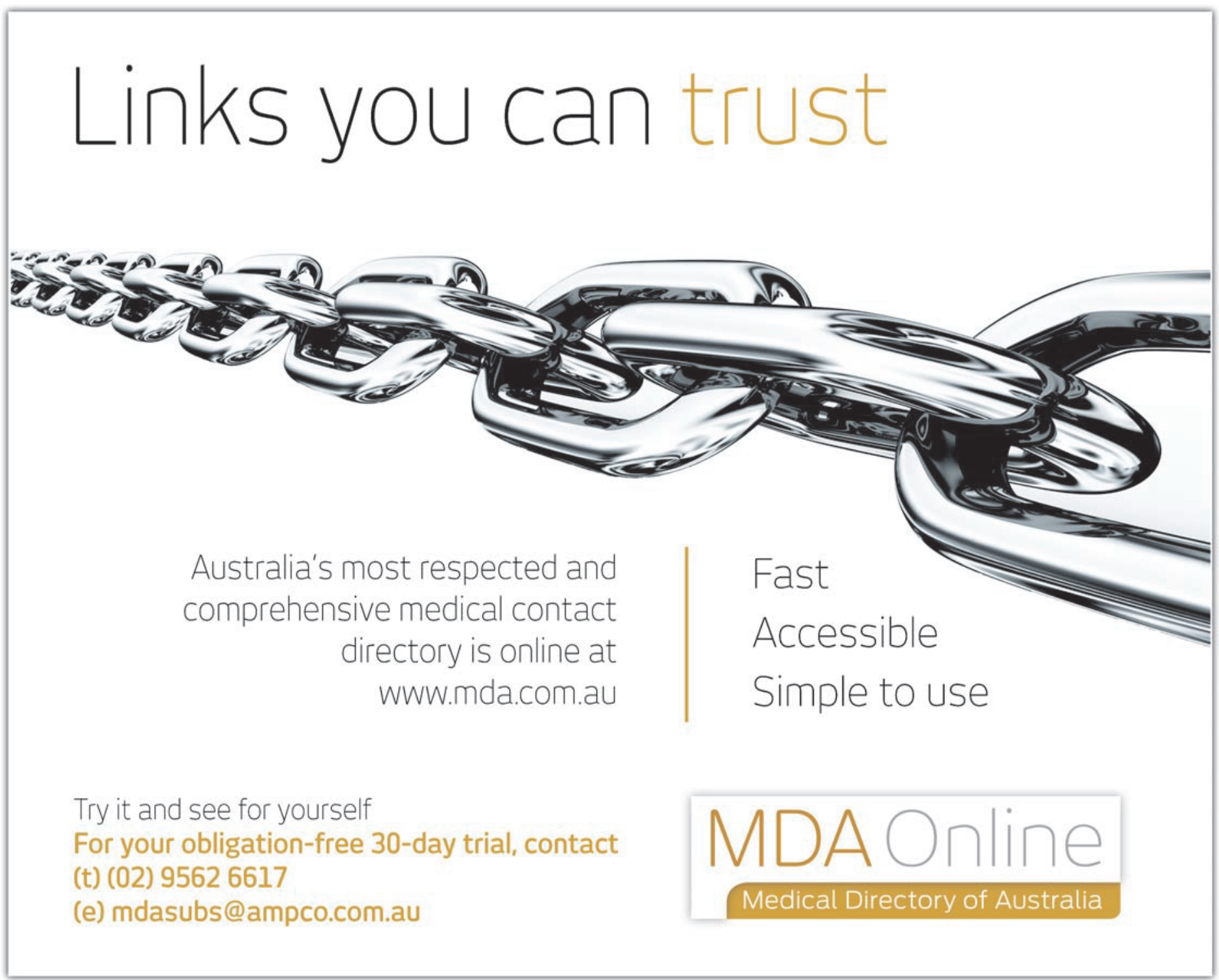

